\title{
Lock-in mechanism of flow over a low-Reynolds-number airfoil with morphing surface
}

\author{
Wei Kang ${ }^{\mathrm{a}}$, Min $\mathrm{Xu}^{\mathrm{a}}$, Weigang Yao ${ }^{\mathrm{b}, *}$, Jiazhong Zhang ${ }^{\mathrm{c}}$ \\ ${ }^{a}$ School of Astronautics, Northwestern Polytechincal University, Shaanxi Province, 710072, \\ P.R.China \\ ${ }^{b}$ School of Mechanical and Aerospace Engineering, Queen's University Belfast, BTr 1NN, \\ $U K$ \\ ${ }^{c}$ School of Energy and Power Engineering, Xi'an Jiaotong University, Shaanxi Province, \\ 710049, P.R. China
}

\begin{abstract}
To understand the frequency lock-in mechanism of flow separation control of an airfoil at low Reynolds number, a systematic analysis is performed by extracting the Lagrangian Coherent Structures (LCSs) from the unsteady flow. The actuation is considered via periodic morphing surface, and the dynamical behaviors between morphing surface and unsteady flow are studied from the viewpoint of fluid transport. Attention is drawn to fluid transport and lift improvement when the actuation frequency is locked onto the vortex shedding frequency. The results show that the fluid particle near the actuator is accelerated by the actuation and interacts with the slow fluid particle in boundary layer on the airfoil surface. The so-called stirring jet mechanism is observed, whereby a cusp structure is formed like a jet acting on the flow, which enhances the fluid transport from main stream into separation zone by reducing dead air zone effectively. The results also show that the actuation frequency is found to be the key factor for lift enhancement and determines the cusp structures and the vortex strength on the upper surface of the airfoil.
\end{abstract}

Keywords: Lock-in; Fluid transport; Flow separation; Lagrangian Coherent Structure; Lift enhancement

\footnotetext{
* Corresponding author

Email address: w.yao@qub.ac.uk (Weigang Yao)
}

Preprint submitted to Aerospace Science and Technology

December 12, 2019 


\section{Nomenclature}

$\lambda_{\max }$ Maximum eigenvalue of Cauchy-Green deformation tensor

$\mu_{\infty} \quad$ Freestream viscosity

$5 \omega \quad$ Flow vorticity

$\phi_{t_{0}}^{t_{0}+T}(\vec{x})$ Map of flow system for LCS extraction

$\rho_{\infty} \quad$ Freestream density

$\sigma \quad$ Finite-time Lyapunov exponents

$(\cdot) \quad$ Dimensional quantity of $(\cdot)$

${ }_{10}(\vec{\cdot}) \quad$ Vector of $(\cdot)$

$A_{0} \quad$ Dimensionless equilibrium amplitude of morphing surface

$A_{m} \quad$ Dimensionless amplitude of morphing motion

c Airfoil chord length

$C_{p} \quad$ Pressure coefficient

${ }_{15} E$ Dimensionless elastic modulus, $E=\frac{\tilde{E}}{\rho U_{\infty}^{2}}$

$f_{\text {excit }}$ Dimensionless frequency of morphing motion

$f_{r e f} \quad$ Dimensionless reference frequency of morphing motion

$L \quad$ Chordwise length of the morphing surface

$R \quad$ Lift ratio of the airfoil with morphing surface and the rigid airfoil

$20 \quad t \quad$ Dimensionless time

$T_{\text {excit }}$ Dimensionless excitation time period 


$\begin{array}{ll}U_{\infty} & \text { Freestream velocity } \\ w & \text { Displacement of morphing surface } \\ w_{0} & \text { Equilibrium position of morphing surface } \\ x^{5} & \text { Dimensionless coordinate } \\ \text { Re } & \text { Reynolds number } \\ \text { ALE } & \text { Arbitrary Lagrangian Eulerian framework } \\ \text { CBS } & \text { Characteristics Based Split scheme } \\ \text { FTLE } & \text { Finite-time Lyapunov exponents } \\ \text { LCS } & \text { Lagrangian Coherent Structure } \\ \text { MAV } & \text { Micro Air Vehicle } \\ \text { UAV } & \text { Unmanned Air Vehicle }\end{array}$

\section{Introduction}

The unmanned aerial vehicles (UAVs) and micro aerial vehicles (MAVs)

35 are ubiquitous and have increasing significance in commercial and military applications [1, such as surveillance, communication relay links, and detection. However, these aerial vehicles are featured with small length scale and low speed, and thus resulting in a low Reynolds number flight environment (i.e. $R e=10^{3} \sim 10^{5}$ ), whereby separated and vortical flow leads to low lift and 40 poor thrust efficiency mainly due to strong viscous effect. Flow control techniques have been developed to manipulate the boundary layer and delay the flow separation, and therefore are desirable to improve aerodynamic performance of UAVs and MAVs.

The small length scale and low Reynolds number flight characteristics render 45 the flow control techniques including slats, flaps [2], which are mainly designed 
for conventional aircraft, less attractive for MAVs. These characteristics further restricted the weight and energy consumption of the actuation system significantly. Therefore, the control techniques such as blowing system and plasma actuator are not feasible for the flow control of MAVs. Inspired by bio-flight, flexible wing offers an alternative flow control strategy, which takes the advantage of the aeroelastic effect of flexible wing made of thin-walled structure with large deflection during the flight. It is expected that the deformation of the flexible structure can adjust the aerodynamic shape to improve the aerodynamic performance. Shyy's group primarily focused on the aerodynamics and aeroelasticity of MAVs since Smith and Shyy computed a flexible membrane airfoil for aerodynamic performance improvement at Reynolds number $R e=4000$ [3]. Excellent reviews on flexible and flapping wing of MAVs can be found in [4, 5] from viewpoint of aerodynamics. Taylor et al. [6] revealed that the interaction between the flexible structure and flow delays the stall and increases the lift significantly by investigating aerodynamic performance of a flexible nonslender delta wing experimentally. Lian et al. 7 presented a CFDbased optimization for the membrane wing design. Gordnier et al. 8] employed a high-order CFD method to further study the fine scale vortical features during the interaction between the flow and flexible wing. Recently, Kang et al. ${ }_{65}$ [9, 10, 11] proposed a locally flexible airfoil model, whereby the flexible structure passively interacts with low Reynolds number flow. As the research on flexible wing is further explored, the studies on flexible structure has been extended in aeronautical engineering. Majić et al. 12 demonstrated an adaptive morphing inlet for turbofan-engine aerodynamic performance improvement. Su et al. 13, 14, studied vibration control and loads improvement for high aspect ratio wings by considering structural flexibility. Burdette et al. 15 highlighted the potential of adaptive morphing trailing edge for the improvement of fuel efficiency of commercial aircraft using aerostructural optimization method. In another recent work by Dan et al. [16], the state-of-art machine learning technique was adopted to optimize the morphing parameters of UAV wing. The aforementioned numerical and experimental work shows great potentials of flex- 
ible structures for aerodynamic performance enhancement. Notably, the results [10, 11, 17, pointed out that the flow structures associated with the actuation frequency have a crucial impact on aerodynamic performance.

From Lagrangian viewpoint, active flow control is to alter a natural flow state or path into a desired state (or path) by manipulating momentum and energy transport of fluid system via external energy from an actuation. This idea paves a way to reveal dynamic features of the coupling system between unsteady flow and actuation systems, which can be used to evaluate the efficiency of flow control strategy. Recent work in dynamical system theory led to the development of tools for fluid transport analysis. As the flow is time-independent or timeperiodic, the stable and unstable manifolds of fixed point or periodic orbits in the flow are the boundaries of the transport and mixing of fluids between different flow regions. Furthermore, fluid transport between different flow regions can be revealed by lobe dynamics, which can be visualized by the tangling between stable manifolds and unstable manifolds. Wiggins et al. [18, 19, 20 investigated the transport and vortex shedding in the near wake of a circular cylinder by using invariant manifolds theory, where the fluid transport in the near wake of a circular cylinder is quantitatively described by lobe dynamics. However, the invariant manifolds theory in their work was not applicable for transient dynamical system in finite time, since the invariant manifolds are defined in time domain $[-\infty,+\infty]$. To circumvent this restriction, Haller and Yuan [21] introduced the concept of finite-time manifolds, i.e., Lagrangian coherent structures (LCSs), which can be used to define the boundaries of transport in finite-time flow. Shadden et al. 22] proposed a mathematical definition of LCSs using finite-time Lyapunov exponents (FTLEs) for two-dimensional aperiodic flows. Eldredge and Chong 23 used LCSs to study the flow pattern of steadily translating and flapping foils, which connects the evolution of attracting LCS with force generation qualitatively. Haller 24] presented a comprehensive review on LCSs and its applications showing that the LCSs are the transport boundaries, and powerful for analyzing transport and mixture of periodic or aperiodic flow. Chen et al. 25] investigated the leading edge vortex dynamics of plunging air- 
foil using LCSs. It is found that distinct flow patterns in LCSs are associated with the formation of leading edge vortex in the slow and fast plunging motion, respectively.

In present study, the frequency lock-in mechanism of active flow control by morphing surface is investigated from viewpoint of fluid transport in Lagrangian framework. The influence of control actuation frequency on aerodynamic performance and the flow separation patterns are highlighted. The paper is organized as follows: Section 2 introduces the numerical formulation of the flow control system. Section 3 describes the definition of Lagrangian Coherent Structures for fluid transport analysis. A systematic analysis of the frequency lock-in mechanism and effects of periodic actuation on fluid transport and separation is provided in Section 4. Conclusions are drawn in Section 5.

\section{Numerical Methodology}

\subsection{Problem set-up}

Fig. 1 and Fig. 2 show the schematic of the problem set-up and computational grid for active flow control by morphing surface, respectively. A local coordinate is introduced by setting $x^{\prime}$ axis along the chord of the morphing part, which is located at the leading edge of the upper surface at $x^{\prime} \in[0,0.1]$ along chordwise direction as shown in Fig. 1. The length of the morphing surface is referred to the dynamic deformed airfoil leading edge 26. The vibration of the morphing surface consists of the initial equilibrium position of the airfoil surface and a periodic motion superimposed on it, which is written as,

$$
\tilde{w}=\tilde{w}_{0}+\tilde{A}_{m} \sin \left(2 \pi \tilde{f}_{\text {excit }} \tilde{t}\right) \sin \left(\frac{\pi x^{\prime}}{\tilde{l}}\right),
$$

130

where $\tilde{l}$ is the length of the morphing surface along the local coordinates, $\tilde{w}$ is the oscillating displacement vertical to the chord at time $\tilde{t} . \tilde{w}_{0}=\tilde{A}_{0} \sin \left(\frac{\pi x^{\prime}}{\tilde{l}}\right)$, $\tilde{A}_{m}$ and $\tilde{f}_{\text {excit }}$ are the equilibrium, amplitude and frequency of the morphing surface, respectively. 


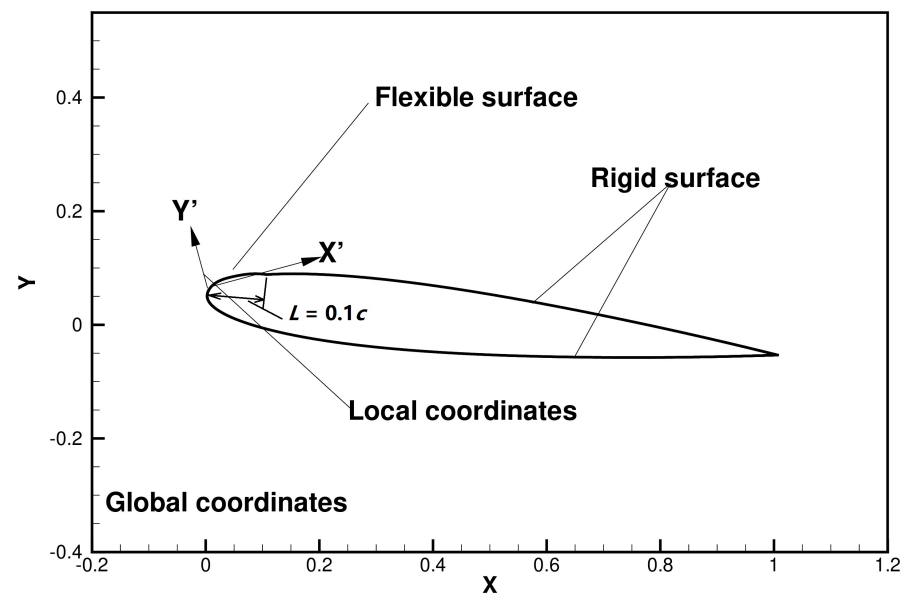

Figure 1: The problem set-up for active flow control by morphing surface.

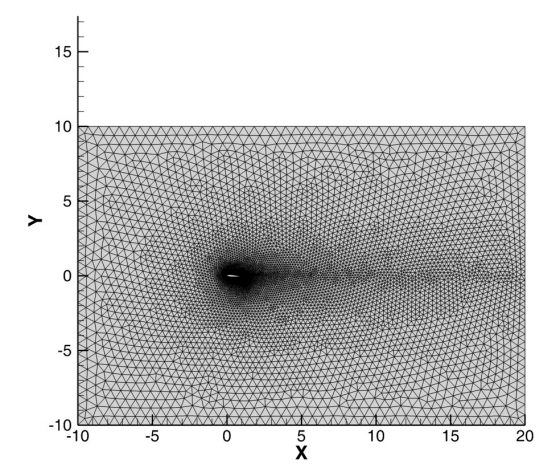

(a) Full domain discretization

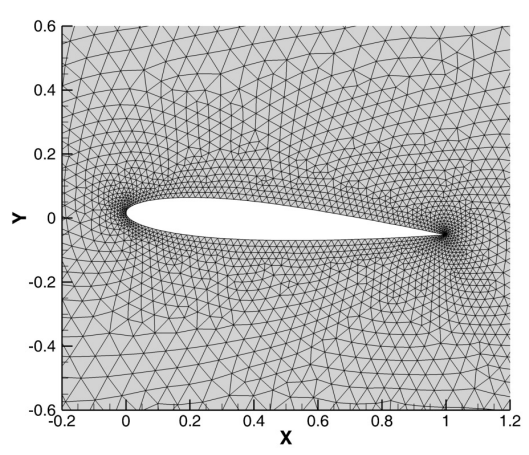

(b) Close-up view of the morphing surface

Figure 2: A representative computational mesh for the flow domain. 
By choosing chord length of the airfoil $c$ as the characteristic scale, and are defined as,

$$
\begin{array}{r}
l=\tilde{l} / c, x=G\left(x^{\prime}\right) / c, w=\tilde{w} / c, w_{0}=\tilde{w}_{0} / c, A_{0}=\tilde{A}_{0} / c, A_{m}=\tilde{A}_{m} / c, \\
f_{\text {excit }}=c \tilde{f}_{\text {excit }} / U_{\infty}, t=U_{\infty} \tilde{t} / c
\end{array}
$$

where $G\left(x^{\prime}\right)$ stands for the transform from local coordinate to global one.

By substituting Eq. (2) to Eq. (1), the vibration of the actuation is converted into the following dimensionless form,

$$
w=w_{0}+A_{m} \sin \left(2 \pi f_{e x c i t} t\right) \sin \left(\frac{\pi x}{l}\right) .
$$

\subsection{ALE-CBS algorithm for unsteady viscous flow}

To study the active flow control, we consider $\vec{x}=(x, y)^{T} \in \Omega_{t} \subset R^{2}$ at time $t \in(0, T)$ as the spatial domain, and the governing equations for two-dimensional unsteady incompressible flow in arbitrary Lagrangian Eulerian (ALE) reference frame are used to solve fluid system and written as,

$$
\left\{\begin{array}{l}
\nabla \cdot \vec{u}=0 \\
\frac{\partial \vec{u}}{\partial t}+\left(\vec{u}-\vec{u}_{g}\right) \nabla \vec{u}=-p+\frac{1}{R e} \nabla^{2} \vec{u}
\end{array}\right.
$$

where $\vec{u}, p$ and $\vec{u}_{g}$ are the fluid density, fluid velocity and the ALE mesh velocity. The Reynolds number $R e=\frac{\rho_{\infty} U_{\infty} c}{\mu_{\infty}}, \rho_{\infty}$ and $\mu_{\infty}$ are the density and dynamic viscosity of the freestream, respectively.

The boundary condition for the actuation in this problem is described as,

$$
\vec{u}=\vec{u}_{a}, \quad \Gamma_{g} \subset \partial \Omega_{t},
$$

where $\vec{u}_{a}$ is the velocity of actuation on the coupling boundary $\Gamma_{g}$.

A finite element method based on Characteristics Based Split scheme under ALE framework (ALE-CBS algorithm) is developed as a fluid solver to obtain aerodynamic characteristics of the airfoil with periodic morphing surface. The 
method is a split procedure by introducing new coordinates along the characteristics, where the convective terms can be eliminated by the coordinate transformation. The resulting equations are only diffusion equations, which can be solved efficiently by standard finite element method. The algorithm can be referred to [27, 28, 10] for more details. Herein only the split procedure of the algorithm is given.

I. Prediction for intermediate velocities.

$$
\begin{aligned}
\vec{U}^{*}-\vec{u}^{n} & =-\Delta t\left[\left(\vec{u}-\vec{u}_{g}\right) \nabla \vec{U}^{n}+\frac{1}{R e} \nabla^{2} \vec{u}^{n}\right] \\
& +\frac{\Delta t^{2}}{2}\left(\vec{u}-\vec{u}_{g}\right) \nabla\left(\left(\vec{u}-\vec{u}_{g}\right) \nabla(\vec{U})^{n}+\frac{1}{R e} \nabla^{2} \vec{u}^{n}\right) .
\end{aligned}
$$

II. Solve the continuity equation implicitly.

$$
\nabla^{2} p^{n}=\frac{1}{\Delta t}\left[\theta_{1} \nabla \cdot \vec{U}^{*}+\left(1-\theta_{1}\right) \nabla \cdot \vec{u}^{n}\right],
$$

where $\theta_{1}$ is relaxation factor. In this case, $\theta_{1}=1$.

III. Correct the velocities with obtained pressure.

$$
\vec{u}^{n+1}-\vec{U}^{*}=-\Delta t \nabla p^{n} .
$$

Eqs. (6)- (8) are the temporal discretization form of NS equations with ALECBS scheme. To solve the NS equations, a linear shape function is adopted for the spatial discretization of fluid velocities and pressure. Spring analogy method [29, 30] is adopted to update the ALE mesh.

In the present study, the computational domain is discretized by unstructured triangular elements and the Reynolds number is chosen as $R e=5000$ [10]. In order to quantify lift enhancement for the airfoil with morphing surface, lift variation ratio $R$ is defined as,

$$
R=\frac{C_{\text {Lexcit }}}{C_{\text {Lrigid }}}
$$

where $C_{L}$ is lift coefficient, the subscript 'excit' denotes the airfoil with morphing surface, whereas 'rigid' denotes the rigid airfoil. 


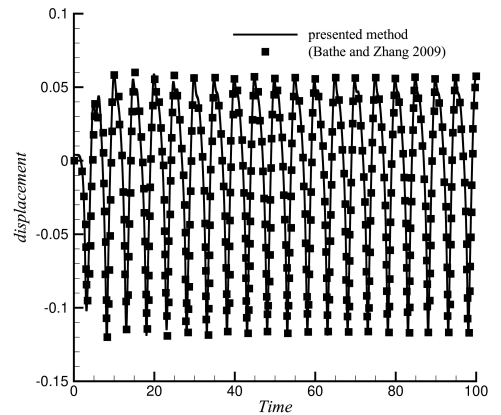

(a) Open-cavity with flexible bottom

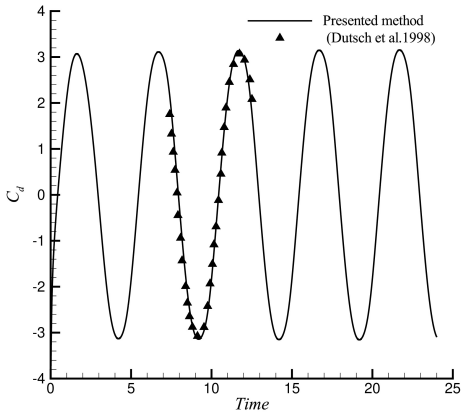

(b) Harmonic in-line oscillation

Figure 3: Validation of ALE-CBS algorithm for fluid-structure interaction.

\subsection{Numerical Methodology Validation}

165

\section{Lagrangian Coherent Structures (LCSs)}

Considering a time-dependent velocity field of fluid particles $\vec{u}(\vec{x}, t)$ defined on an open set $D \subset R^{2}$, the trajectory starts at point $\vec{x}_{0} \in D$ at time $t_{0}$. According to the definition of fluid particle velocity, the velocity field $\vec{u}$ is written as,

$$
\begin{aligned}
& \overrightarrow{\dot{x}}=\vec{u}(\vec{x}, t), \\
& \vec{x}\left(t_{0}, \vec{x}_{0}\right)=\vec{x}_{0} .
\end{aligned}
$$

The solution of the system defined in Eq. 10 can be viewed as a map, denoted by $\phi^{t}{ }_{t_{0}}$, and satisfies

$$
\phi_{t_{0}}^{t}: D \rightarrow D: \vec{x}_{0} \mapsto \phi_{t_{0}}^{t}\left(\vec{x}_{0}\right)=\vec{x}\left(t, \vec{x}_{0}\right) .
$$


FTLEs is used to delineate the attracting or repelling structures in finite-time interval $\left[t_{0}, t_{0}+T\right]$, which is defined by the maximum eigenvalue of corresponding Cauchy-Green deformation tensor given in Eq. 12.

$$
\sigma_{t_{0}}^{T}(\vec{x})=\frac{1}{|T|} \ln \sqrt{\lambda_{\max }\left(\left(\frac{d \phi_{t_{0}}^{t_{0}+T}(\vec{x})}{d \vec{x}}\right)^{*} \frac{d \phi_{t_{0}}^{t_{0}+T}(\vec{x})}{d \vec{x}}\right)},
$$

where ()$^{*}$ denotes the transpose of the tensor, $\sigma_{t_{0}}^{T}(\vec{x})$ stands for the FTLEs, $\phi_{t_{0}}^{t_{0}+T}(\vec{x})$ is the map of flow system. Accordingly, the repelling LCSs are depicted by the ridges of FTLE field with $T>0$, and the attracting LCSs are depicted by the ridges of FTLE field with $T<0$.

In this study, the motions of the fluid particles are computed by the ALECBS algorithm shown in Eqs (6)-(8). The velocity field $\vec{u}(\vec{x}, t)$ is interpolated using bicubic splines onto a fine structure quadrilateral mesh $(1000 \times 500$ in $x \in[0,1.5], y \in[-0.2,0.2])$ in the region near the airfoil. Trajectories of the passive tracers are integrated by solving Eq. 10 with fourth order RungeKutta method. FTLEs are computed via the Cauchy-Green deformation tensor, in which the derivative of the flow map is approximated by central difference scheme. FTLEs then can be visualized by contour plot for Lagrangian analysis of unsteady flow.

\section{Results and discussions}

\subsection{Effect of actuation frequency on lift enhancement}

The reference position $A_{0}=0.00365$ and amplitude of the morphing motion $A_{m}=0.00222$ were chosen according to the primary mode shape and amplitude of elastic structure with dimensionless elastic modulus $E=5 \times 10^{4}$, which achieves highest lift enhancement among passive flow control cases of locally flexible structure [10. The effect of actuation frequency is of particular interest as it has great impact on aerodynamic performance and flow evolution [33, 34, 35. Herein, actuation frequencies are selected as $m \times f_{\text {ref }}, m=0.3 \cdots 2$, where 
the reference oscillating frequency $f_{\text {ref }}=1.3570$ is the primary frequency of the structure with dimensionless elastic modulus $E=5 \times 10^{4}$.

The variation ratio $R$ for lift and lift-drag ratio is plotted in Fig. 4 as a function of actuation frequency. The result suggests that the lift and lift-drag ratio of the airfoil with local periodic morphing surface is improved except in the case of $f_{\text {exit }} / f_{\text {ref }} \approx 0.3$. Specifically, the lift increases approximately more than $20 \%$ in the frequency range $0.5<f_{\text {exit }} / f_{\text {ref }}<1.4$, where the frequency lockin appears [36, 37]. The peak is found at $f_{\text {exit }} / f_{\text {ref }} \approx 0.9$ with approximately $69.86 \%$ lift improvement. However, the lift enhancement becomes marginal for $f_{\text {exit }} / f_{\text {ref }}>1.5$ indicating that the effect of actuation decreases significantly. In the lock-in region, the vortex shedding frequency begins to synchronize with the actuation frequency $f_{\text {exit }}$ at the lock-in onset $\left(f_{\text {excit }} / f_{\text {ref }} \approx 0.5\right)$ and gradually recovers to its rigid airfoil counterpart as the actuation frequency increases, which is elucidated in Fig. 5 by the first two dominate frequencies of the flow. Notably, the primary frequency of the flow is locked on to the actuation frequency, while the second frequency is twice higher than the primary frequency in the lock-in region. As the actuation frequency further increases to $f_{\text {excit }} / f_{\text {ref }}>1.4$, the primary frequency recovers to the frequency of its rigid airfoil counterpart, whereas the second frequency recovers to the actuation frequency, and the lift enhancement gradually deteriorates and ceases at $f_{\text {excit }} / f_{\text {ref }} \approx 2.8$. To further understand the lock-in mechanism, flow patterns are investigated by LCSs at $f_{\text {excit }} / f_{\text {ref }}=0.5,0.9,1.3,2.0$ from the fluid transport viewpoint in the next section.

\subsection{Effect of actuation frequency on fluid transport}

In this section, a set of four representative actuation frequencies is chosen to elucidate the frequency lock-in regime from the fluid transport viewpoint. Fig. 6 shows the FTLE fields of the airfoil at four representative actuation frequencies. It is worth noting that it is not sufficient to use only a few level sets of FTLE field to determine the LCSs. According to LCSs definition introduced by Shadden [22], the LCSs are the ridges of FTLE field, which are defined as the zero level 


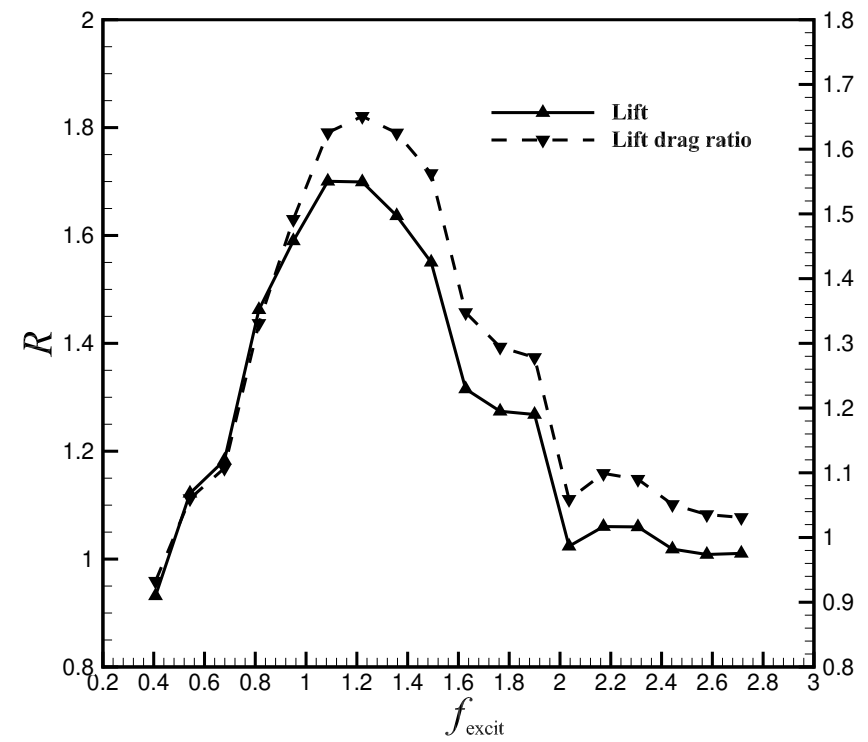

Figure 4: Lift and lift-drag ratio of the airfoil as a function of the actuation frequency (Length of Morphing surface $=0.1 c$ ).

set of inner product of FTLE gradient and eigenvector of FTLE Hessian matrix corresponding to the smallest eigenvalue. However, the ridges are considered to be adequate as shown in Fig. 6 in the present study. The approach was also used in [38, 39].

Attracting LCSs can be viewed as a boundary dividing the flow into two regions, namely, the main stream and separation zone. It was well understood that the fluid transport can be manifested by the tangling between the repelling and attracting LCSs [40. For periodic flow, the repelling and attracting LCSs are steady except near the trailing edge, where repelling and attracting LCSs tangle together and vortices shed alternately into the flow wake, which suggests no fluid transport exists until the trailing edge. In Fig. 6 (a)-(e), the socalled "dead air zone", where no fluid transport exists, is shaded in green color. Fig. 6 (b) shows that repelling LCSs fold toward the main stream and start to tangle with the attracting LCSs at $f_{\text {excit }} / f_{\text {ref }} \approx 0.5$. A vortex is deduced as a 


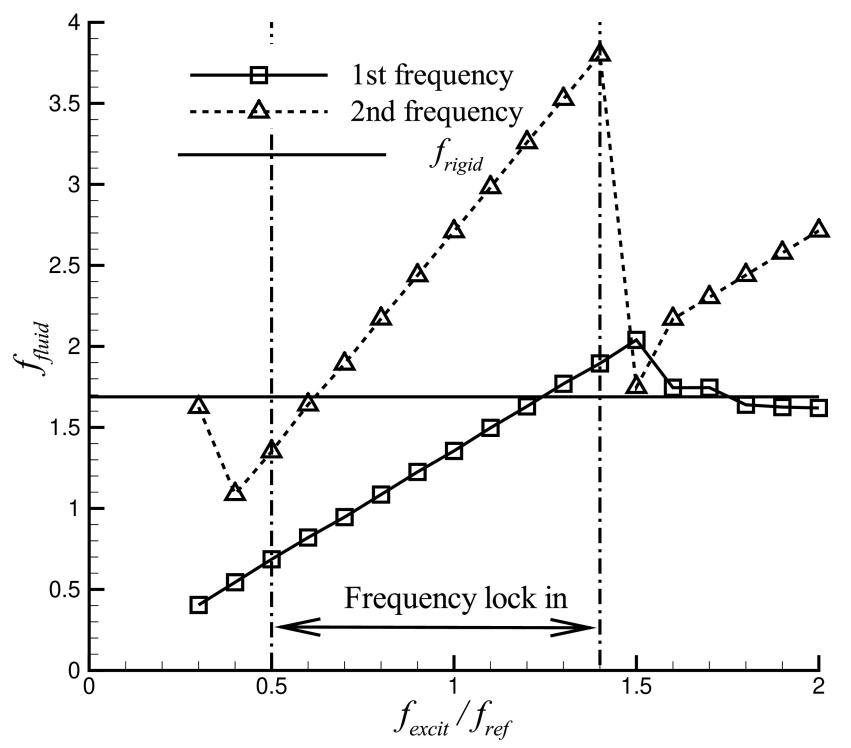

Figure 5: The first two frequencies of the main stream as a function of the actuation frequency.

result on the middle of the upper surface of the airfoil, moves downstream and eventually sheds into the wake with a growing size. In addition, the dead air zone size is reduced compared with the rigid airfoil counterpart, which suggests that the fluid transport from the main stream into the dead air zone starts to be enhanced by the actuation at $f_{\text {excit }} / f_{\text {ref }} \approx 0.5$. This is also evident from pressure distribution, as shown in Fig. 7(b), where the pressure on the upper surface becomes lower than rigid airfoil and the resulting lift is enhanced. It is expected that the pressure fluctuates due to the vortices alternately shedding near the trailing edge of the airfoil.

The smallest dead air zone is achieved at $f_{\text {excit }} / f_{\text {ref }} \approx 0.9$ as shown in Fig. 6 (c), whereby both the fluid transport and the lift are most enhanced due to the tangling between the attracting and repelling LCSs. This is further confirmed by the lower pressure on the upper surface of the airfoil in Fig. 7 (c). This observation indicates that the lift amplification interlinks with the size of the dead air zone. In other words, the lift enhancement increases as the size of 
dead air zone decreases. Similar to $f_{\text {excit }} / f_{\text {ref }}=0.5$, a vortex is deduced as a regime, the dead air zone is reduced, and fluid transport is enhanced due to the tangling between the two types of LCSs. The highest lift amplification occurs at $f_{\text {excit }} / f_{\text {ref }} \approx 0.9$, where the actuation frequency synchronizes with 
the frequency of the main stream and the tangling effect is most pronounced as well as the fluid transport. As the frequency further increases and the lock-in terminates, the tangling deteriorates gradually and the lift recovers to the rigid airfoil. To further generalize our finding, we next examine the flow separation pattern under actuation.

\subsection{Effect of the actuation on flow separation}

In this section, the influences of the actuation on separation pattern are elucidated. Fig. 8 shows time history of separation and reattachment points at the same four representative frequencies $f_{\text {excit }} / f_{\text {ref }}=0.5,0.9,1.3,2.0$ as the section 4.2 The separation and reattachment points are computed from instantaneous velocity field and identified by the following equation,

$$
\left.\omega\right|_{\left(x_{0}, y_{0}\right)}=0, \frac{\partial \omega}{\partial \vec{n}} \begin{cases}>0, & \text { separation position, } \\ <0, & \text { reattachment position. }\end{cases}
$$

where $\omega$ is the vorticity at point $\left(x_{0}, y_{0}\right), \vec{n}$ is the unit normal vector to the airfoil surface.

As shown in Fig. 8 (a), the separation point of the rigid airfoil is at $x \approx$ 0.3745 , and reattachment point is near the trailing edge and oscillates due to alternate vortex shedding. It is evident from the figure that the number of separation bubbles increases as the frequency increases from 0.5 to 2.0 , and the size of the bubbles decreases in contrast, which is further elucidated by the cusp structures in Fig. 6. In addition, the frequency of the birth of the separation bubble is locked onto the actuation frequency in the lock-in regime ${ }_{10}\left(0.9<f_{\text {excit }} / f_{\text {ref }}<1.3\right)$ and recovers to the frequency of main stream at $f_{\text {excit }} / f_{\text {ref }} \approx 2.0$. The dash line in Fig. 8 also suggests that the horizontal velocity (velocity in $x$ direction) of the separation bubble remains unchanged as the actuation frequency increases from 0.9 to 2.0. To further discern the separation pattern, the amplitude of the fluctuation pressure near the trailing edge is used to demonstrate the strength of the separation bubbles on the upper surface of the airfoil. Fig. 7 shows that the strength of the separation bubbles 
reaches the peak at $f_{\text {excit }} / f_{\text {ref }} \approx 0.9$, where the lift enhancement also acquires the maximum. As the actuation frequency $f_{\text {excit }} / f_{\text {ref }}$ further increases from 1.3 to 2.0 , the strength of the separation bubbles reduces significantly as well as the amplitude of the fluctuation pressure near the trailing edge, and the lift recovers to the rigid airfoil.

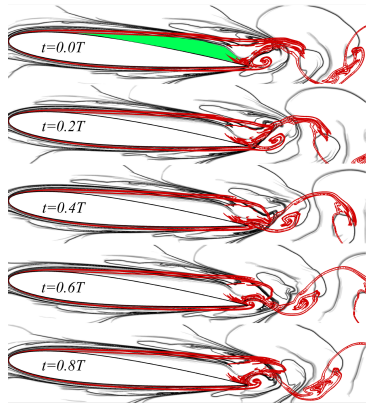

(a) Rigid airfoil

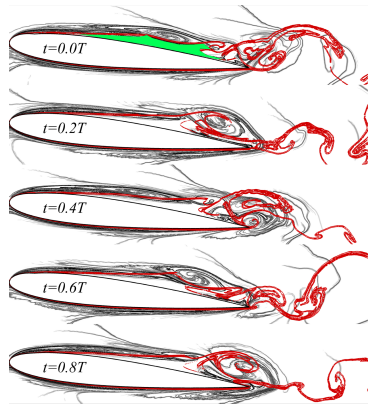

(b) $f_{\text {excit }} / f_{\text {ref }}=0.5$

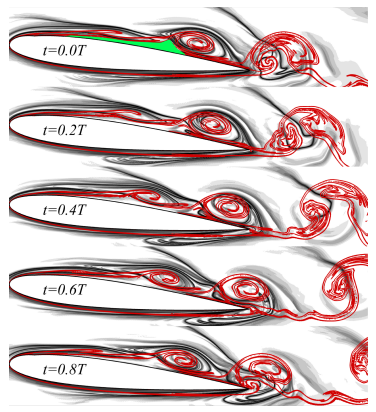

(c) $f_{\text {excit }} / f_{\text {ref }}=0.9$

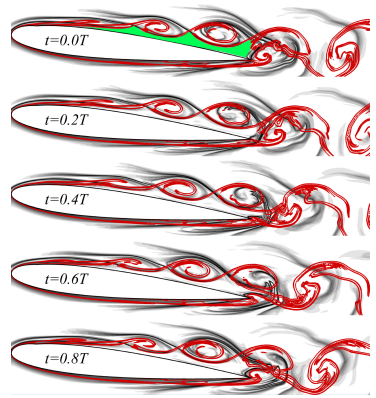

(d) $f_{\text {excit }} / f_{\text {ref }}=1.3$

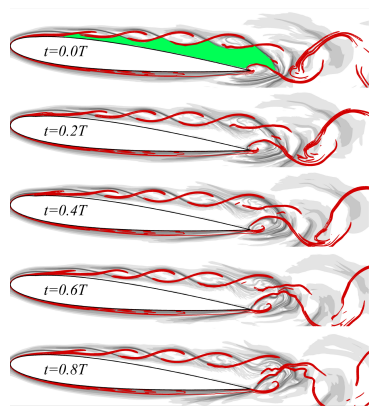

(e) $f_{\text {excit }} / f_{\text {ref }}=2.0$

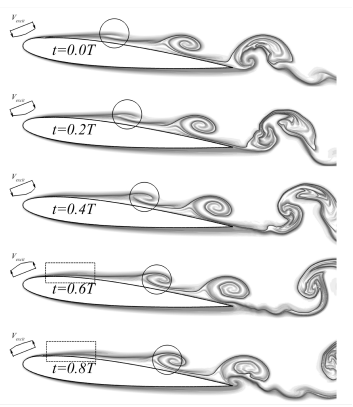

(f) Attracting LCSs with $f_{\text {excit }} / f_{\text {ref }}=0.9$

Figure 6: FTLE fields at the four representative actuation frequencies. ((a)-(e):Repelling LCSs:Grayscale; Attracting LCSs: Red.)

\section{Conclusions}

To explore effective active flow control techniques, an airfoil with a periodic morphing surface is considered in this study. The control mechanism is studied 
actuation frequency on lift enhancement is emphasized. Based on the systematic study, the following conclusion can be drawn.

(i) The lift is enhanced at $0.5<f_{\text {exit }} / f_{\text {ref }}<1.40$, where the lock-in occurs. The highest lift enhancement acquires at $f_{\text {exit }} / f_{\text {ref }} \approx 0.9$, where the frequency of the main flow synchronizes with the actuation frequency.

(ii) The tangling between attracting and repelling LCSs defines the dead air zone, which determines fluid transport and the lift enhancement. As the tangling between the two types of LCSs is pronounced, the dead air zone is reduced and the lock-in appears. The two types of LCSs tend to overlap as the actuation increases further to $f_{\text {exit }} / f_{\text {ref }}>2.0$, where the lock-in terminates. Cusp structures are observed and act like stirring jet flow, which eventually evolve into separation bubble.

(iii) The number of separation bubbles increases as the actuation frequency increases. However, the actuation frequency has no influence on the horizontal velocity of the separation bubbles.

Our future work is to find the connection between the stirring jet and fluid transport via lobe dynamics, quantitatively.

\section{Acknowledgement}

The research is supported by the National Natural Science Foundation of China (Grant No. 11972307), the Fundamental Research Funds for the Central Universities (Grant No. 3102017zy039), Top International University Visiting Program for Outstanding Young scholars of Northwestern Polytechnical University.

\section{References}

[1] T. J. Mueller, J. D. DeLaurier, Aerodynamics of small vehicles, Annual Review of Fluid Mechanics 35 (1) (2003) 89-111. 
[2] S. Scott Collis, R. D. Joslin, A. Seifert, V. Theofilis, Issues in active flow control: theory, control, simulation, and experiment, Progress in Aerospace Sciences 40 (4) (2004) 237-289.

[3] R. Smith, W. Shyy, Computation of unsteady laminar flow over a flexible two-dimensional membrane wing, Physics of Fluids 7 (9) (1995) 2175-2185.

[4] W. Shyy, M. Berg, D. Ljungqvist, Flapping and flexible wings for biological and micro air vehicles, Progress in Aerospace Sciences 35 (5) (1999) 455505.

[5] W. Shyy, H. Aono, S. Chimakurthi, P. Trizila, C. Kang, C. Cesnik, H. Liu, Recent progress in flapping wing aerodynamics and aeroelasticity, Progress in Aerospace Sciences 46 (7) (2010) 284-327.

[6] G. Taylor, Z. Wang, E. Vardaki, I. Gursul, Lift enhancement over flexible nonslender delta wings, AIAA Journal 45 (12) (2007) 2979-2993. doi: 10.2514/1.31308.

[7] Y. Lian, W. Shyy, D. Viieru, B. Zhang, Membrane wing aerodynamics for micro air vehicles, Progress in Aerospace Sciences 39 (6-7) (2003) 425-465.

[8] R. E. Gordnier, S. Kumar Chimakurthi, C. E. Cesnik, P. J. Attar, Highfidelity aeroelastic computations of a flapping wing with spanwise flexibility, Journal of Fluids and Structures 40 (2013) 86-104.

[9] W. Kang, J. Z. Zhang, P. H. Feng, Aerodynamic analysis of a localized flexible airfoil at low reynolds numbers, Communications in Computational Physics 11 (4) (2012) 1300-1310.

[10] W. Kang, J. Z. Zhang, P. F. Lei, M. Xu, Computation of unsteady viscous flow around a locally flexible airfoil at low reynolds number, Journal of Fluids and Structures 46 (2014) 42-58.

[11] P. F. Lei, J. Z. Zhang, W. Kang, S. Ren, L. Wang, Unsteady flow separation and high performance of airfoil with local flexible structure at low reynolds number, Communications in Computational Physics 16 (3) (2014) 699-717. 
[12] F. Majić, G. Efraimsson, C. J. O’Reilly, Potential improvement of aerodynamic performance by morphing the nacelle inlet, Aerospace Science and Technology 54 (2016) 122-131.

[13] J. R. Hammerton, W. Su, G. Zhu, S. S.-M. Swei, Optimum distributed wing shaping and control loads for highly flexible aircraft, Aerospace Science and Technology 79 (2018) 255-265.

[14] N. Tsushima, W. Su, A study on adaptive vibration control and energy conversion of highly flexible multifunctional wings, Aerospace Science and Technology 79 (2018) 297-309.

[15] D. A. Burdette, J. R. Martins, Design of a transonic wing with an adaptive morphing trailing edge via aerostructural optimization, Aerospace Science and Technology 81 (2018) 192-203.

[16] D. Xu, Z. Hui, Y. Liu, G. Chen, Morphing control of a new bionic morphing uav with deep reinforcement learning, Aerospace Science and Technology.

[17] G. Papadakis, M. Santer, G. Jones, Control of low reynolds number flow around an airfoil using periodic surface morphing: a numerical study, Journal of Fluids and Structures 76 (2018) $95-115$.

[18] J. Duan, S. Wiggins, Lagrangian transport and chaos in the near wake of the flow around an obstacle: a numerical implementation of lobe dynamics,

nonlinear Processes in Geophysics 4 (3) (1997) 125-136. doi:10.5194/ npg-4-125-1997.

[19] N. Malhotra, S. Wiggins, Geometric structures, lobe dynamics, and lagrangian transport in flows with aperiodic time-dependence, with applications to rossby wave flow, Journal of nonlinear science 8 (4) (1998) 401-456.

[20] S. Wiggins, The dynamical systems approach to lagrangian transport in 
[21] G. Haller, G. Yuan, Lagrangian coherent structures and mixing in twodimensional turbulence, Physica D: Nonlinear Phenomena 147 (3) (2000) $352-370$.

[22] S. C. Shadden, F. Lekien, J. E. Marsden, Definition and properties of lagrangian coherent structures from finite-time lyapunov exponents in twodimensional aperiodic flows, Physica D: Nonlinear Phenomena 212 (3) (2005) 271-304.

[23] J. D. Eldredge, K. Chong, Fluid transport and coherent structures of translating and flapping wings, Chaos: An Interdisciplinary Journal of Nonlinear Science 20 (1) (2010) 017509.

[24] G. Haller, Langrangian coherent structures, Annual Review of Fluid Mechanics 47 (1) (2015) 137-162.

[25] J. Chen, J. Zhang, S. Cao, Using lagrangian coherent structure to understand vortex dynamics in flow around plunging airfoil, Journal of Fluids and Structures 67 (2016) $142-155$.

[26] M. Sahin, L. N. Sankar, M. Chandrasekhara, C. Tung, Dynamic stall alleviation using a deformable leading edge concept-a numerical study, Journal of aircraft 40 (1) (2003) 77-85.

[27] O. C. Zienkiewicz, R. Codina, A general algorithm for compressible and incompressible flow .1. the split, characteristic-based scheme, International Journal for Numerical Methods in Fluids 20 (8-9) (1995) 869-885.

[28] O. C. Zienkiewicz, K. Morgan, B. V. K. S. Sai, R. Codina, M. Vasquez, A general algorithm for compressible and incompressible-flow .2. tests on the explicit form, International Journal for Numerical Methods in Fluids 430 20 (8-9) (1995) 887-913.

[29] J. T. Batina, Unsteady euler algorithm with unstructured dynamic mesh for complex-aircraft aerodynamic analysis, AIAA Journal 29 (3) (1991) 327-333. 
[30] F. J. Blom, Considerations on the spring analogy, International Journal for Numerical Methods in Fluids 32 (6) (2000) 647-668.

[31] K. J. Bathe, H. Zhang, A mesh adaptivity procedure for cfd and fluidstructure interactions, Computers \& Structures 87 (11) (2009) 604-617.

[32] H. Dütsch, F. Durst, S. Becker, H. Lienhart, Low-reynolds-number flow around an oscillating circular cylinder at low keulegan-carpenter numbers, Journal of Fluid Mechanics 360 (1998) 249-271.

[33] D. Greenblatt, I. J. Wygnanski, The control of flow separation by periodic excitation, Progress in Aerospace Sciences 36 (7) (2000) 487-545.

[34] M. Amitay, A. Glezer, Controlled transients of flow reattachment over stalled airfoils, International Journal of Heat and Fluid Flow 23 (5) (2002) 690-699.

[35] A. Glezer, M. Amitay, A. M. Honohan, Aspects of low-and high-frequency actuation for aerodynamic flow control, AIAA journal 43 (7) (2005) 15011511.

[36] J. Young, J. C. Lai, Vortex lock-in phenomenon in the wake of a plunging airfoil, AIAA journal 45 (2) (2007) 485-490.

[37] D. Raveh, E. Dowell, Frequency lock-in phenomenon for oscillating airfoils in buffeting flows, Journal of Fluids and Structures 27 (1) (2011) 89-104.

[38] D. Lipinski, B. Cardwell, K. Mohseni, A lagrangian analysis of a twodimensional airfoil with vortex shedding, Journal of Physics A: Mathematical and Theoretical 41 (34) (2008) 344011.

[39] S. Cao, Y. Li, J. Zhang, Y. Deguchi, Lagrangian analysis of mass transport and its influence on the lift enhancement in a flow over the airfoil with a synthetic jet, Aerospace Science and Technology 86 (2019) 11 - 20. 
[40] H. Salman, J. S. Hesthaven, T. Warburton, G. Haller, Predicting transport by lagrangian coherent structures with a high-order method, Theoretical and Computational Fluid Dynamics 21 (1) (2007) 39-58. 


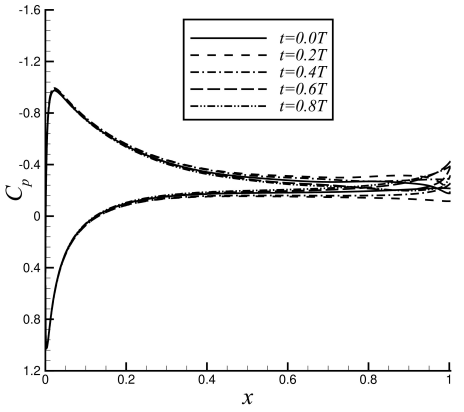

(a) Rigid airfoil

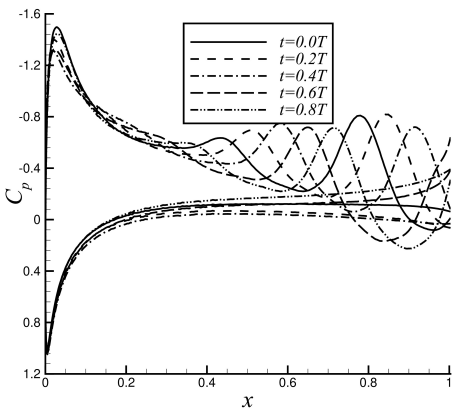

(c) $f_{\text {excit }} / f_{\text {ref }}=0.9$

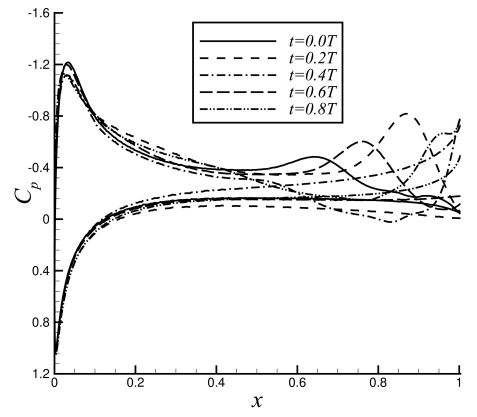

(b) $f_{\text {excit }} / f_{\text {ref }}=0.5$

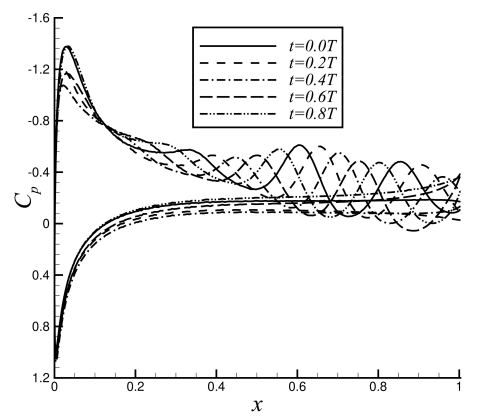

(d) $f_{\text {excit }} / f_{\text {ref }}=1.3$

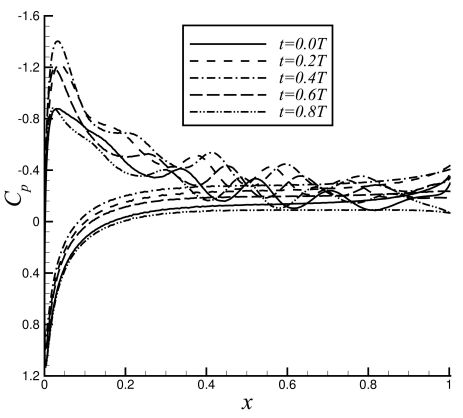

(e) $f_{\text {excit }} / f_{\text {ref }}=2.0$

Figure 7: Pressure distribution at the four representative actuation frequencies. 


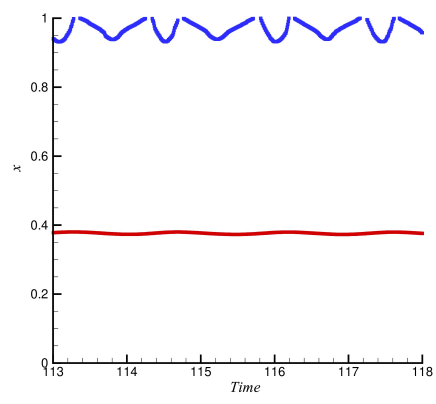

(a) Rigid airfoil

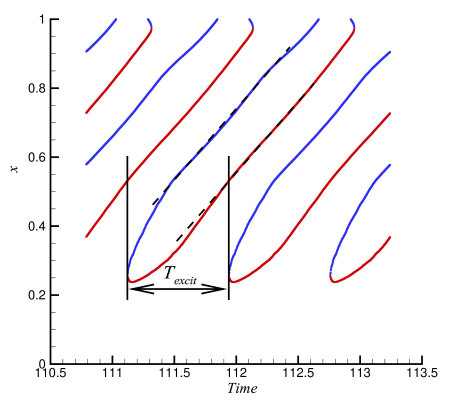

(c) $f_{\text {excit }} / f_{\text {ref }}=0.9$

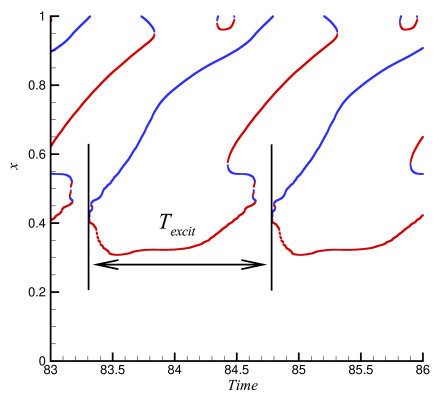

(b) $f_{\text {excit }} / f_{\text {ref }}=0.5$

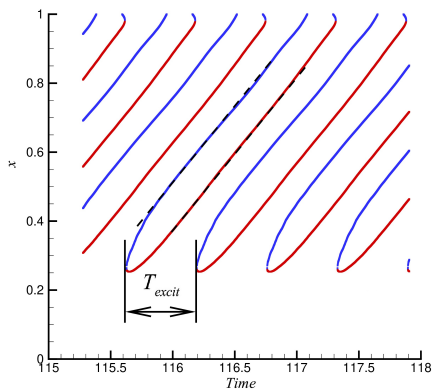

(d) $f_{\text {excit }} / f_{\text {ref }}=1.3$

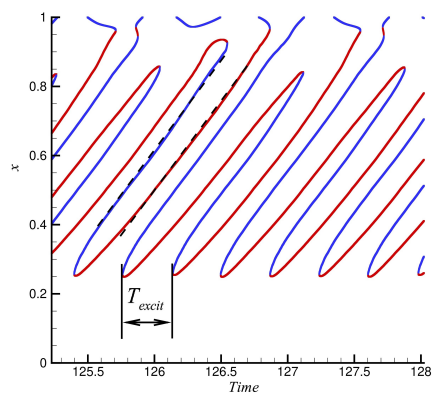

(e) $f_{\text {excit }} / f_{\text {ref }}=2.0$

Figure 8: Time history of separation and reattachment points at the four representative actuation frequencies. (RED: Separation position; BLUE: Reattachment position) 